\title{
Failure Rate Analysis of Boeing 737 Brakes Employing Neural Network
}

\author{
Ahmed Z. Al-Garni ${ }^{1}$, Ahmad Jamal ${ }^{2}$, Farooq Saeed ${ }^{3}$, Ayman H. Kassem ${ }^{4}$ \\ King Fahd University of Petroleum and Minerals, Dhahran, 31261, Saudi Arabia
}

\begin{abstract}
The failure rate analysis of brake assemblies of a commercial airplane, i.e., Boeing 737, is analyzed using the artificial neural network and Weibull regression models. One-layered feed-forward back-propagation algorithm for artificial neural network whereas three parameters model for Weibull are used for the analysis. Three years of data are used for model building and validation. The results show that the failure rate predicted by neural network is closer in agreement with the actual data than the failure rate predicted by the Weibull model. Results also indicate that neural network can be effectively integrated into an aviation maintenance facility computerized material requirement planning system to forecast the number of brake assemblies needed for a given planning horizon.
\end{abstract}

\section{Nomenclature}

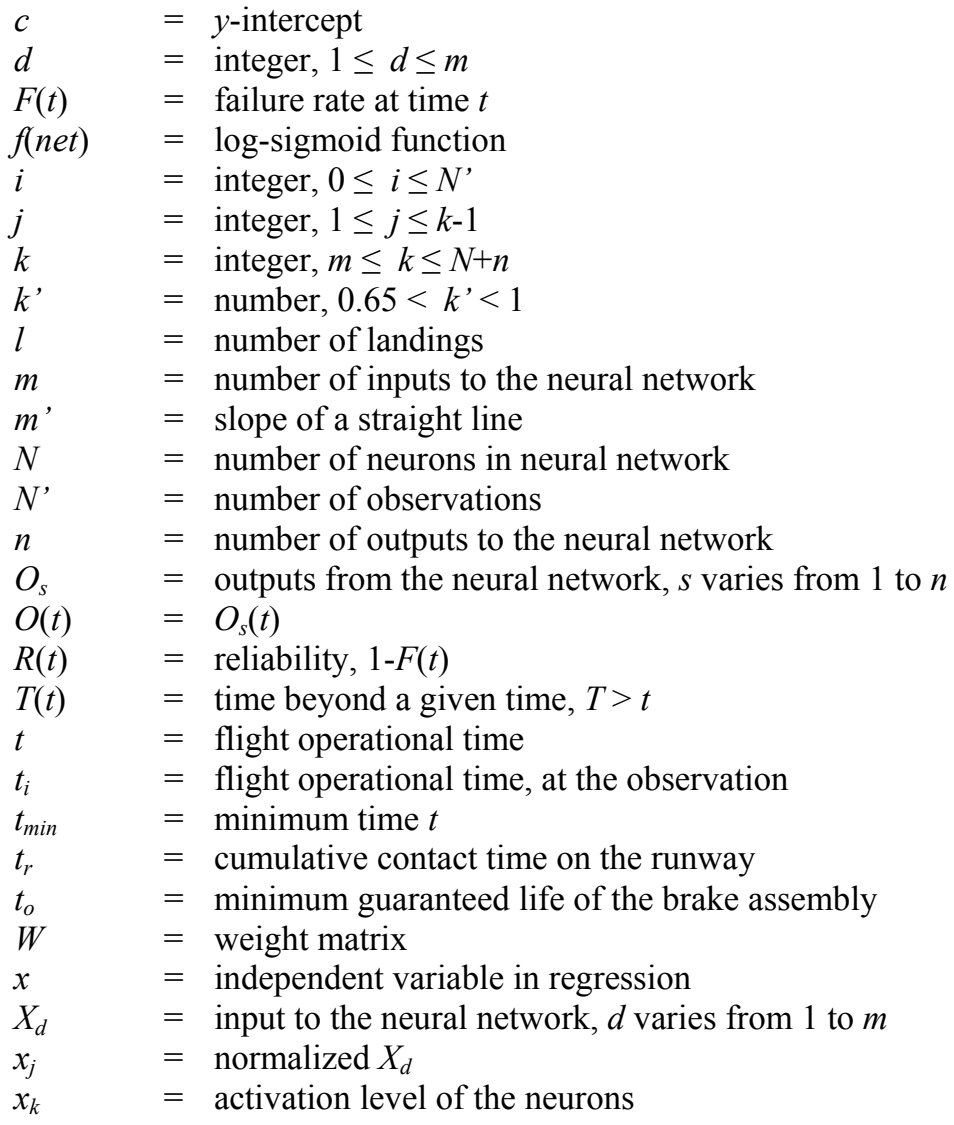

${ }^{1}$ Professor, Aerospace Engineering Department, P. O. Box 842.

${ }^{2}$ Lecturer, Aerospace Engineering Department, P. O. Box 1066.

${ }^{3}$ Assistant Professor, Aerospace Engineering Department, P. O. Box 1637.

${ }^{4}$ Assistant Professor, Aerospace Engineering Department, P. O. Box 873. 
$y=$ dependent variable in regression

$\beta, \eta \quad=$ parameters of Weibull model

$\lambda(t) \quad=$ instantaneous failure rate of the brake assembly

\section{Introduction}

Airplanes such as the Boeing 737 are repairable systems that include several non-repairable parts; brake assemblies are among the non-reparable parts that must be replaced upon wear/failure. The Boeing 737 is an American aircraft ${ }^{1,2}$ with an operating mass (empty) of $27,955 \mathrm{~kg}$, a maximum payload mass of $15,136 \mathrm{~kg}$, an overall length of $30.53 \mathrm{~m}$, an overall height of $11.28 \mathrm{~m}$, a wing area of $91.04 \mathrm{~m}^{2}$, a wing span of $28.35 \mathrm{~m}$, a wing chord (at root) of $4.71 \mathrm{~m}$, a maximum cruising speed (at altitude 10,060 m) of $462 \mathrm{knots}(856 \mathrm{~km} / \mathrm{h}$ ), a range (for 115 passengers) of 1855 nautical miles $(3437 \mathrm{~km})$, and an approximate take-off field length of $2000 \mathrm{~m}$. It uses a brake unit with four rotor multiple-disc-type brakes as shown in Fig. 1. The location and environment ${ }^{3}$ in which the aviation fleet operates are mostly in the Eastern Province of the Arabian Peninsula. The climate in the Province is influenced by the Arabian Gulf waters. Dhahran is one of its main cities. The weather conditions in most main cities are more or less the same. Dhahran $(26.32 \mathrm{~N}, 50.13 \mathrm{E})$ can be selected as having representative weather for the Eastern Province, which is nearly $1 \mathrm{~km}$ inland from the Gulf. In the past ten years, the monthly average temperature has varied from $15^{\circ} \mathrm{C}$ to $38^{\circ} \mathrm{C}$, the monthly average humidity from $34 \%$ to $75 \%$, and the monthly average solar radiation from 320 to $560 \mathrm{~W} . \mathrm{h} / \mathrm{m}^{2} /$ day. A proper record of wear/failure data is valuable in interpreting the wear/failure pattern, for comparative evaluation of the quality of brake assemblies of various manufacturers and for prediction of future needs in a specified planning horizon or for specified operational hours.

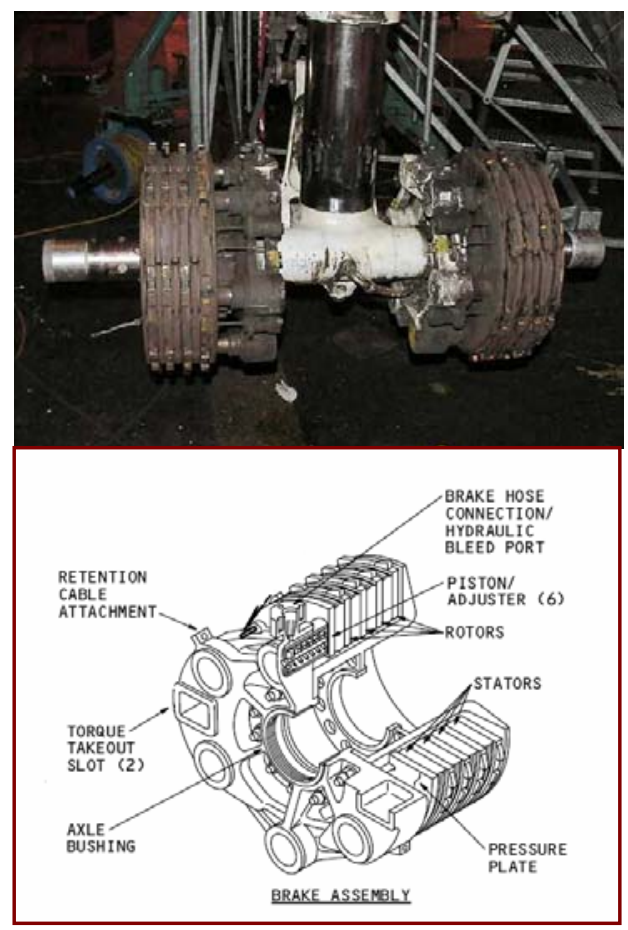

Figure 1. Brake assembly of Boeing 737 airplane.

Airplane brakes are subjected to a number of wear-out processes, i.e., uniform wear, accelerated wear at certain spots, micro chipping, etc. When the brakes are applied upon landing, the conditions of wear in airplanes are far more severe than the corresponding conditions in automobiles on the highways. In the case of airplanes, the loads are not so uniform. There are varieties of shock loads or a severe load spectrum is generated, which can cause accelerated wear. Brake life is defined by the wear limits set by the controlling aviation agencies. When the damage 
due to these wear-out processes reaches this critical limit, the brake assembly is considered to be worn out/failed. Replacement of the brakes is due to wear/failure. The indicator pin of the brake assembly will indicate its wear limit depending on factory-imposed limits. However, the brake assembly can be replaced for other reasons, e.g., overheating of the brake assembly. The brake assembly absorbs a tremendous amount of heat energy and whenever it shows signs of overheating or if it has been involved in an aborted take-off, it must be removed from the airplane and given a complete inspection following, which it may be replaced. Chattering or squealing will generate vibration, which is harmful to the landing gear and brake structure. Warped or glazed discs will cause chattering as will any unparallel condition of the surface of the disc stack.

The time taken to reach this critical manifestation of wear can be measured either by the associated flight time or in terms of the number of landings. Let us consider a situation where the flight time $t$ is proportional to the time of application of the airplane brakes on the runway, $t_{r}$, which in turn is proportional to the number of landings, $l$. It can be written as:

$$
t \propto t_{r} \text { and } t \propto l
$$

The brake assembly life is not a fixed value but rather a random quantity in terms of time, $t$ or number of landings, $l$, and is bounded by $t_{o}<t<\infty$ or $l_{o}<l<\infty$, respectively where $t_{o}$ and $l_{o}$ are the minimum expected lives in terms of time (hours) and number of landings, respectively also referred to as safe lives.

Modeling the failure rate of airplane brakes accurately is of prime interest. This model should accurately predict the time of brake failure in order to avoid crashes during landing or take-off. Various conventional regression models can be developed to model this failure rate. However, recently, a lot of interest has been focused on the application of Artificial Neural Network (ANN) in modeling. ${ }^{411}$ It is eminent from the previous work that the failure rate prediction model for the brake assembly has not been developed for Boeing 737 . The objective of the present work is to develop an ANN model that predicts the failure rate of Boeing 737 airplane brake assemblies based on flight operational time in addition to employing the data in Weibull regression model that has been used in the past in the aerospace, automotive, and manufacturing industries. Furthermore, the predicting capabilities of both models are also demonstrated. The rest of the paper is organized as follows: in section 2, the failure data for the brake assemblies in terms of flight operational time in hours is presented; in section 3, the ANN and the Weibull regression model are developed; a comparison of the results obtained from the ANN and Weibull model with the actual data is presented in section 4 ; and section 5 concludes the paper.

\section{Brakes Failure Data}

The data was collected from a local aviation facility in Saudi Arabia. The data represents the failure data of brake assemblies for Boeing 737 over a period of three years for a fleet of four airplanes. These four airplanes have the registration numbers N737A, N739A, N743A, and N745A. Data was collected for brake assemblies installed on each of the four man landing gears. Therefore, there are four brake assemblies, two on the left and two on the right. The present analysis focuses on the brake assemblies rather than airplanes. The reason being that the airplane brakes are subjected to same operational conditions, i.e., climatic, runway, and loading conditions, therefore, it is more important and useful to develop the model for the brake assemblies over the four airplanes. Brakes are numbered as 1 and 2 on the right, and 3 and 4 on the left of the airplanes as shown in Fig. 2. Thus $B_{1}$ refers to the first brake assembly outboard on the right main landing gear. Similarly $B_{3}$ refers to the third brake assembly inboard on the left main landing gear. Failure is defined whenever, at the inspection time, it is observed that the brake assembly needs to be replaced according to the aviation standards being followed. The data, which is obtained from the logbook of each airplane, are recorded in two forms, i.e., as flying time in hours between the replacements and as number of landings between the replacements. In the present study, flying time is used as indicator of life of the brake assemblies. 

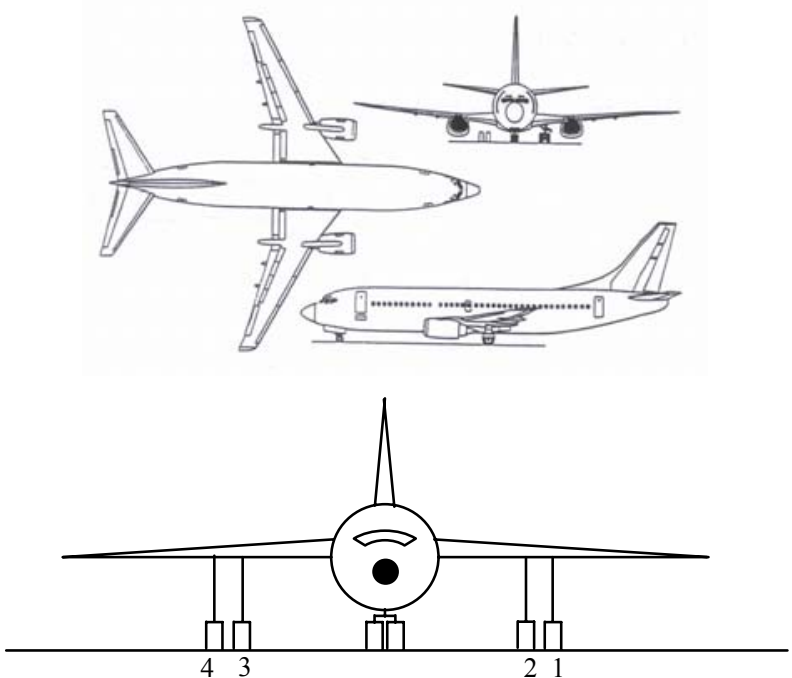

Figure 2. Boeing 737 airplane sketch for four main brake assemblies.

\section{Brakes Failure Prediction Models}

\section{A. Artificial Neural Network (ANN)}

\section{Introduction}

An artificial neural network is an information-processing system that has certain performance characteristics in common with biological neural networks. ANNs are computational systems that mimic the biological neural networks of the mammalian brain. The human brain contains about 100 billion neurons (neuron cells), interconnected in a complex manner via synapses (junctions between axons and dendrites), thus constituting a network. An ANN is a collection of neurons that are arranged in specific formations. Neurons are grouped into layers. A multilayer network usually consists of an input layer, one or more hidden layers, and an output layer. The number of neurons in the input layer corresponds to the number of parameters that are presented to the network as inputs. The same is true for the output layer. ANN analysis is not limited to a single output and neural nets can be trained to build neuron models with multiple outputs. The neurons in the hidden layer or layers are responsible primarily for feature extraction. They provide increased dimensionality and accommodate such tasks as classification and prediction. ${ }^{11}$

\section{Development of ANN}

The basic idea of artificial neural network was initiated by MuClloch and Pitts. ${ }^{12}$ They studied the ability of a model neuron to interconnect several basic components. Later, Rosenblatt ${ }^{13}$ coined the name "perceptron" and devised an architecture that received much attention. However, a rigorous analysis of the perceptron made by Minsky and Papert ${ }^{14}$ demonstrated that it had certain limitations. This almost brought the research in this area to a halt, but later the work of Hopfield ${ }^{15}$ revived the interest in ANN. Since then, a variety of ANN algorithms have been proposed and used in recent years. Presently, research on ANN is being performed in a great number of disciplines ranging from neurobiology and psychology to engineering sciences.

\section{Back-Propagation Algorithm}

Some other algorithms are also in use such as Radial Bases Function neural network (RBF), Recurrent neural network, Hopfield neural network, Self Organizing Map (SOM), etc. ${ }^{16}$ The Back-Propagation (BP) algorithm is among the popular learning algorithms for artificial neural network ${ }^{17-20}$. BP algorithm is the simplest and well known for its good performance. It is in fact a gradient descent-error-correcting algorithm. Before beginning training, some small random numbers are usually used to initialize each weight on each connection. BP requires preexisting training patterns and involves a forward-propagation step followed by a back-propagation step. The forward-propagation step begins by sending the input signals through the nodes of each layer. A non-linear 
activation function, called the sigmoid function, is usually used at each node for the transformation of the incoming signals to an input signal. This process repeats until the signals reach the output layer and an output value is calculated. The back-propagation step calculates the error by comparing the calculated and target outputs. New sets of weights are iteratively calculated by modifying the existing weights based on these error values until a minimum overall error or global error is obtained. The Mean Square Error (MSE) is usually used as a measure of the global error. ${ }^{16}$ The following logic is assumed in back-propagation. ${ }^{17}$

$$
\begin{gathered}
x_{j}=\text { normalized } X_{d} \quad 1<d \leq m \\
n^{2} t_{k}=\sum_{j=1}^{k-1} W_{k j} x_{j}+b_{j} \quad m+1 \leq k \leq N+n \\
x_{k}=f\left(\text { net }_{k}\right) \quad m+1 \leq k \leq N+n \\
O_{s}=x_{N+s} \quad 1 \leq s \leq n \\
f\left(\text { net }_{k}\right)=\frac{1}{1+e^{-n e t_{k}}}
\end{gathered}
$$

$X_{d}$ represents the actual inputs to the ANN (which have to be normalized and then initially stored in $x_{j}$ ). The nonlinear activation function $f\left(\right.$ net $\left._{k}\right)$ in Eq. (5) is log-sigmoid function and it depends on the desired output data range. $N$ is a constant, which represents the number of intermediate neuron in the ANN. It can be any integer as long as it is not less than $m$. The value of $N+m$ determines how many neurons are there in the network (if we include the inputs as neuron). The size of the weight matrix $W$ in each layer depends on the number of neurons in the corresponding adjacent layers of ANN. The term $x_{k}$ is called the "activation level" of the neuron, and $O_{s}$ is the output from ANN. The notational input and output to the neuron and the network design of back-propagation are shown in Fig. 3.
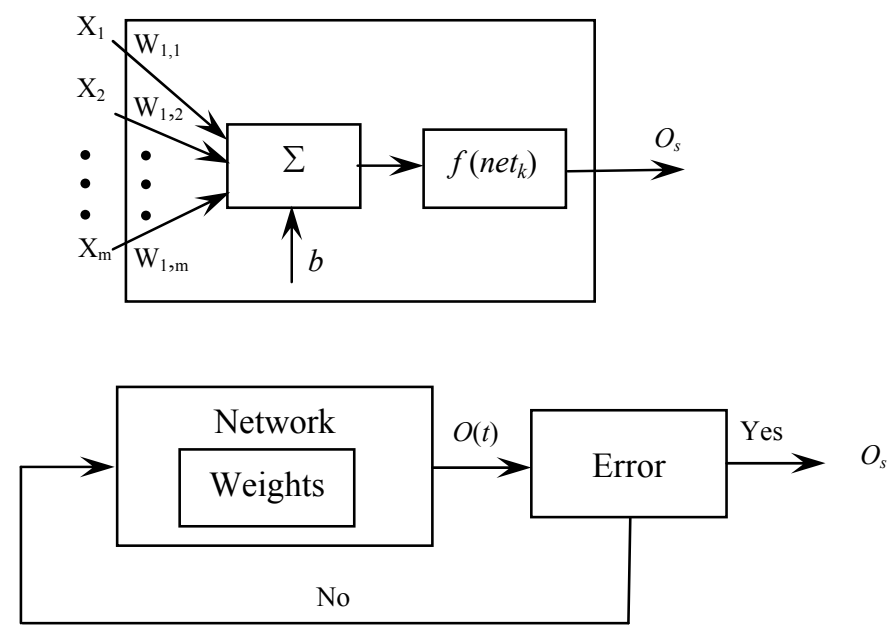

Figure 3. Artificial neuron with activation function and network design of back-propagation.

\section{ANN Model for Present Analysis}

In this section, an artificial neural network is developed to model the failure rate of the brakes. The input to the neural network is time in hours and the output to the ANN is the failure rate corresponding to that time. The activation function (log-sigmoid function) takes the input and squashes the output into the range from 0 to 1 as shown in Fig. 4. This function is commonly used in multi-layer networks that are trained using the back-propagation 
algorithm and also this function is differentiable. The predicted failure rate can be found by using the forward-pass calculation Eqs. (1)-(4). The training of the neural network is carried out using the back-propagation technique. The objective is to minimize the sum squared error give by:

$$
\text { error }=\sum(F(t)-O(t))^{2}
$$

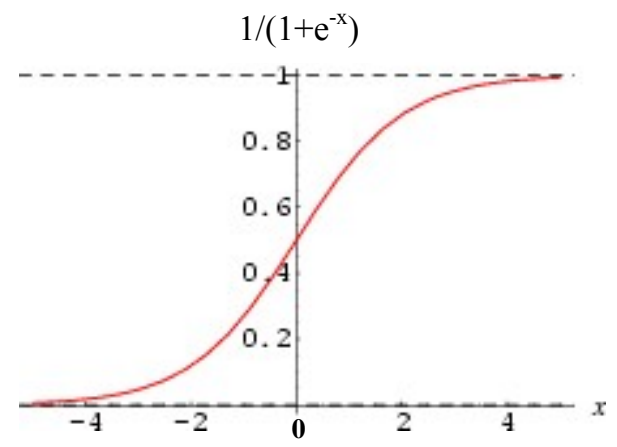

Figure 4. Log-sigmoid function.

Where $F(t)$ is the actual failure rate in terms of time (hours). $O(t)$ is the final output in time (hours), which is calculated from the ANN model. The number of passes is usually set to a high number. The initial error is high because the initial weights were assigned randomly. As the network is trained, the error decreases and converges to a minimum value. Since the present study represents a dynamic system, which is one whose state varies with time, a model known as autoregressive model that uses inputs corresponding to previous points in time can be used. ${ }^{16}$ Therefore, for ANN model selection, only data in terms of time in hours from the same source is taken and following four cases are studied:

1) One input $m=1$, one output $n=1$, and four intermediate neurons $N=4$,

2) Two inputs $m=2$, one output $n=1$, and four intermediate neurons $N=4$,

3) Three inputs $m=3$, one output $n=1$, and four intermediate neurons $N=4$,

4) Four inputs $m=4$, one output $n=1$, and four intermediate neurons $N=4$.

For $2^{\text {nd }}, 3^{\text {rd }}$, and $4^{\text {th }}$ case, one, two and three previous time inputs are taken, respectively, for each time input. The comparison of all four cases is presented in Fig. 5. The average percentage differences of the failure rate with that of the actual brake failure data are found to be $12.25 \%, 8.34 \%, 4.10 \%$, and $3.92 \%$ for ANN having one, two, three, and four inputs, respectively. It is evident from the percentage differences that the ANN results improve as the number of inputs increase but the model with four inputs does not bring drastic improvement in results from that of three inputs. Therefore, three inputs ANN model has been adopted for the present study. 


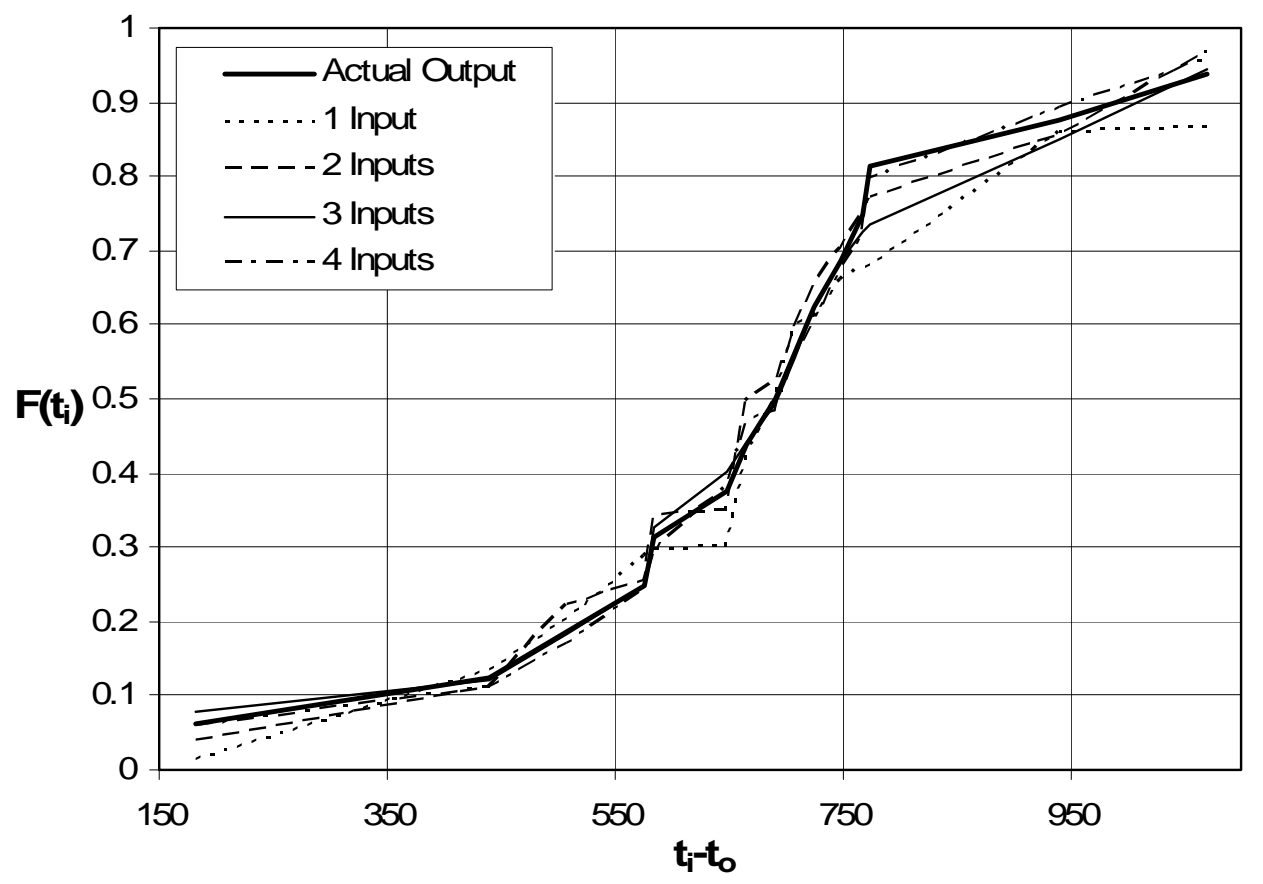

Figure 5. Comparison of failure rate $F\left(t_{i}\right)$ against time, predicted by using 1, 2, 3, and 4 inputs.

Furthermore, the analysis was also extended to study the effect of the number of intermediate neurons as shown in Fig. 6. The percentage differences for two, four, six, ten, and fifteen intermediate neurons came out to be $18.56 \%$, $8.63 \%, 4.60 \%, 4.18 \%$, and $4.11 \%$, respectively. It is obvious from the percentages that little improvement has been achieved by increasing the number of neurons beyond six at the expense of more complexity in the network and program execution time. Hence, six intermediate neurons are selected fro the analysis. The ANN model of the present study uses single intermediate layer of neurons since single layer is commonly used and gives reasonable results. $^{7}$

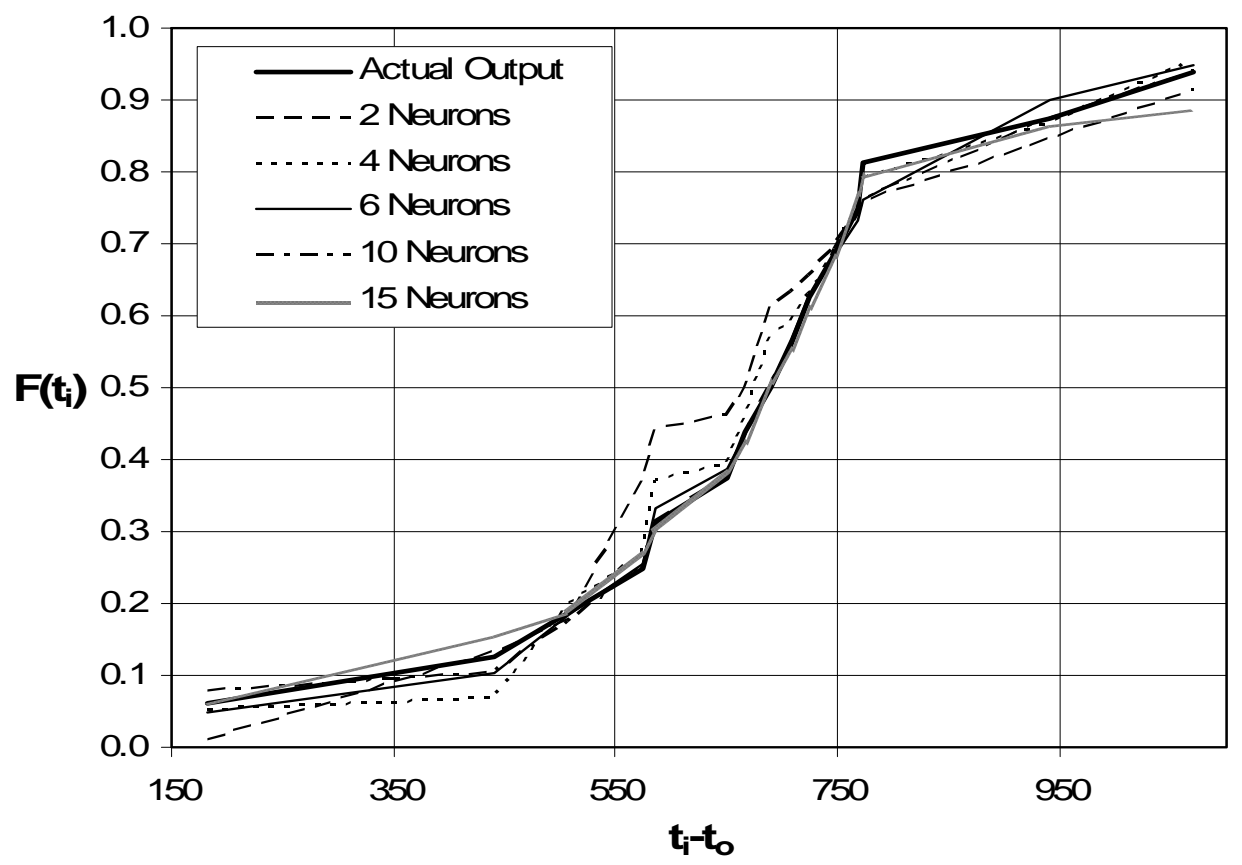

Figure 6. Comparison of failure rate $F\left(t_{i}\right)$ against time, predicted by using 2, 4, 6, 10, and 15 neurons. 
The working flow chart of the entire analysis is shown in Fig. 7 and the ANN architecture employed is shown in Fig. 8. The size of the weight matrices $W_{l}$ and $W_{2}$ are $6 \times 3$ and $1 \times 6$, respectively. Training the back-propagation network requires the following:

1) Select the training pair from the training set; apply the input vector to the network input terminal.

2) Calculate the output of the network (using Eqs. (1)-(4), forward pass).

3) Calculate the error (the difference between the network output and desired output).

4) Adjust the weights of the network in a way that minimizes the error. It would quicken the process if the weights not being used are zeroed out.

5) Repeat steps 1-4 for each vector in the training set until the error for the entire set is acceptably low. Steps 1 and 2 constitute the forward while steps 3 and 4 are the reverse passes.

The above steps can easily be understood by the flow chart shown in Fig. 9 .

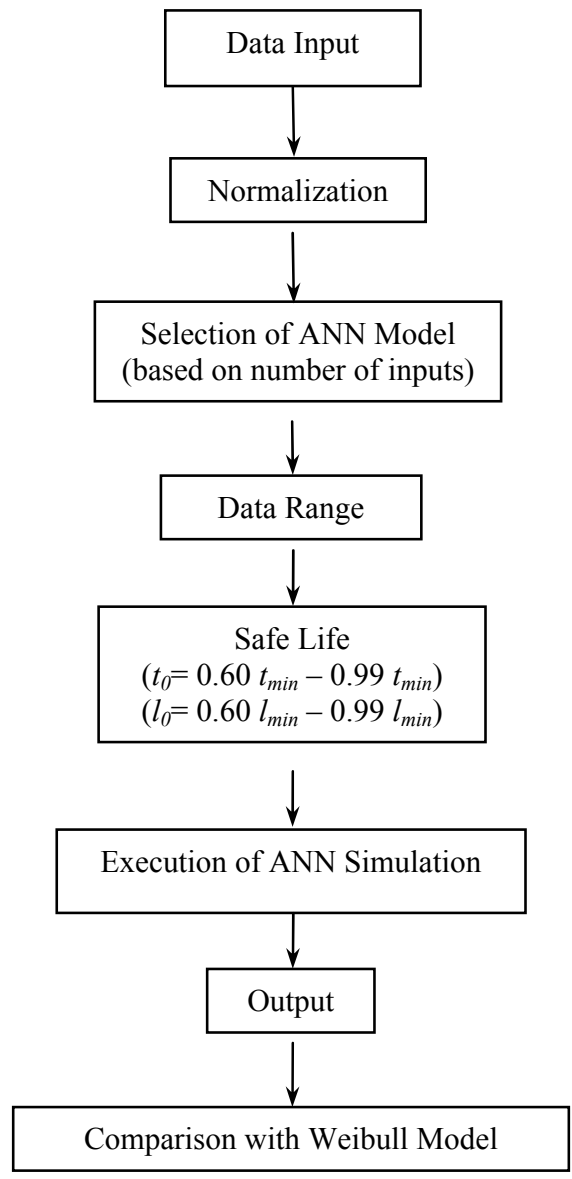

Figure 7. Flow chart of the entire analysis. 


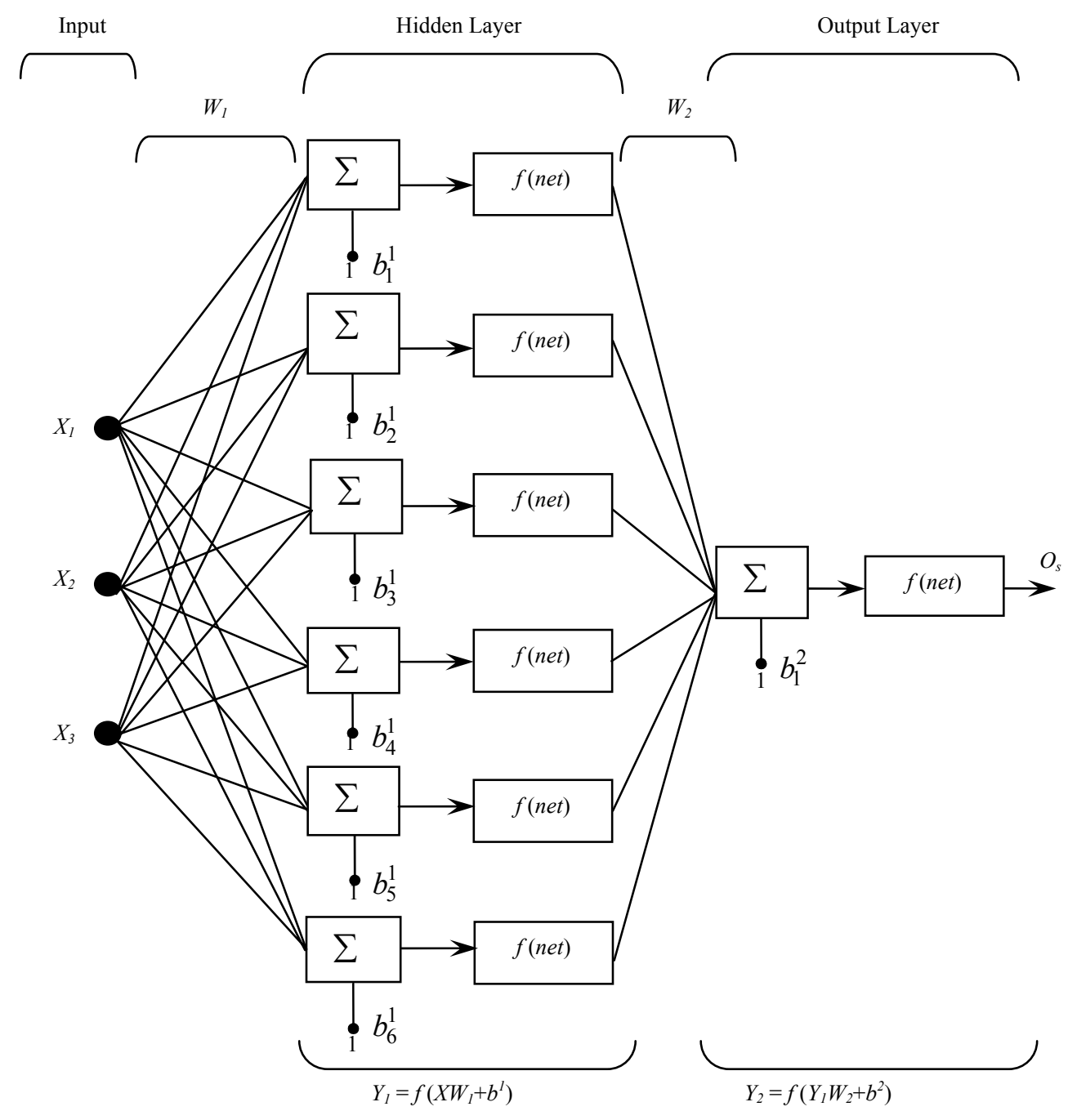

Figure 8. ANN architecture. 


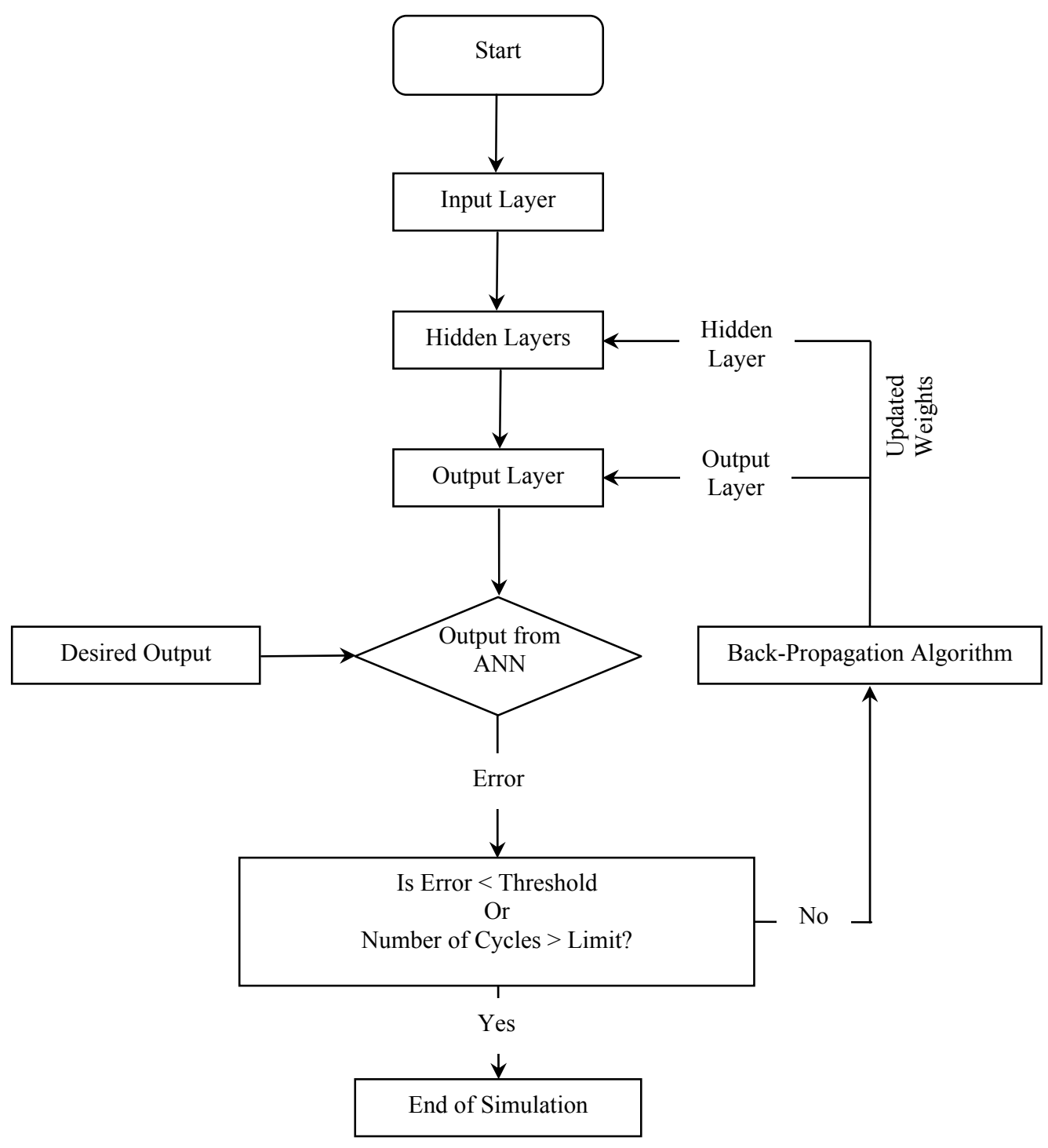

Figure 9. Flow chart of ANN architecture.

\section{B. Weibull Regression Model}

5. Reliability analysis of brake assembly wear/failure data in terms of flight time, $t$

The reliability $R(t)$ of a brake assembly characterizes the probability of its survival beyond a given time $t$, i.e., $R(t)=P(T>t)$, and in general terms, it can be defined as: ${ }^{21,22}$

$$
R(t)=\exp \left[-\int_{0}^{t} \lambda(t) d t\right]
$$

Where $\lambda(t)$ is the instantaneous failure rate of the brake assembly and $t$ is proportional to $t_{r}$, which in turn, is proportional to $l$. Brake assemblies are subjected to an increasing failure rate as the operational time, i.e., the number of landings, increases. Thus the most suitable characterization on instantaneous brake failure rate will be described by a power-law function of time, so that 


$$
\lambda(t)=\frac{\beta}{\eta-t_{0}}\left(\frac{t-t_{0}}{\eta-t_{0}}\right)^{\beta-1}
$$

Where $\eta$ is a scale parameter that expresses the characteristic life and $\beta$ is a shape parameter of the model that determines the severity of the wear-out process. Using this power-law failure rate model, Eqs. (7) and (8) will represent a well known three-parameter Weibull reliability model, which can be written as follows:

$$
R(t)=\exp \left[-\left(\frac{t-t_{0}}{\eta-t_{0}}\right)^{\beta}\right] \quad t>t_{0}
$$

Where $t$ is the random variable characterizing the life of the brake assembly; $t_{0}<t<\infty$. To fit the data, the complementary function to the reliability function $R(t)$ is often used, which is also known as the cumulative function $F(t)=1-R(t)$ and defines $P(T>t)$. Thus using Eq. (9), one can write

$$
F(t)=1-\exp \left[-\left(\frac{t-t_{0}}{\eta-t_{0}}\right)^{\beta}\right] \quad t>t_{0}
$$

$F(t)$ is failure rate at time $t$. Among various approaches used in fitting the Weibull model to the failure data, a procedure used by Sheikh et al. ${ }^{22}$ is the most lucid and easy to implement. This method linearizes the equation as follows:

$$
\begin{gathered}
\ln [1-F(t)]=-\left(\frac{t-t_{0}}{\eta-t_{0}}\right)^{\beta} \\
\ln \left\{\ln \left[\frac{1}{1-F(t)}\right]\right\}=\beta \ln \left(t-t_{0}\right)-\beta \ln \left(\eta-t_{0}\right)
\end{gathered}
$$

Now let

$$
\begin{aligned}
& y=\ln \left[\ln \left(\frac{1}{1-F(t)}\right)\right] \\
& x=\ln \left(t-t_{0}\right) \\
& m^{\prime}=\beta \\
& c=-\beta \ln \left(\eta-t_{0}\right)
\end{aligned}
$$

Equation (11) is now in the form

$$
y=m^{\prime} x+c
$$

Where $x$ and $y$ are the independent and dependent variables in regression, respectively, $m$ is the slope of the plot, and $c$ is the $y$-intercept. After arranging the failure data in ascending order, the probability distribution function can be substituted by its estimate using the median rank formula: ${ }^{21}$

$$
F\left(t_{i}\right)=\frac{i}{N^{\prime}+1} \quad 1 \leq i \leq N^{\prime}
$$

Where $N^{\prime}$ is the number of observations. Linearized Eq. (12) can be fitted to the experimental data $F\left(t_{i}\right)$ versus $\left(t_{i}-t_{0}\right)$ for $i=1,2,3,4, \ldots \ldots, N^{\prime}$. By performing the linear regression analysis using linearly transformed Eq. (12), the parameters $\beta$ and $\eta$ can be determined. This approach implies that $t_{0}$ is known. The value of $t_{0}$ is equal to $k^{\prime} t_{\min }$, where $0.65<k^{\prime}<1$ and $t_{\min }$ is the minimum time $t$. A starting point can be taken as $t_{0}=0.6 t_{\min }$. If a straight line fit 
is poor, then this value can be adjusted between $0.65 t_{\min }$ and $0.99 t_{\min }$ until a good fit is obtained. A spreadsheet (MS Excel) was used to perform this analysis on the brake assemblies of all the four airplanes. Table 1 gives the complete analysis for $B_{4}$. The regression output for this analysis is presented in Table 2, which gives the values of the parameters of the Weibull model. Thus the failure rate model for $B_{4}$ is

$$
F(t)=1-\exp \left[-\left(\frac{t-336.70}{1116.49-336.70}\right)^{2.3762}\right]
$$

Table 1. Regression analysis of the failure data (h) of $B_{4}$ for Boeing 737.

\begin{tabular}{ccccccc}
\hline \hline & & & & & \\
$\boldsymbol{i}$ & $\boldsymbol{t}_{\boldsymbol{i}}(\mathbf{h})$ & $\boldsymbol{X}_{\boldsymbol{d}}=\left(\boldsymbol{t}_{\boldsymbol{i}}-\boldsymbol{t}_{\boldsymbol{~}}\right)$ & $\ln \left(\boldsymbol{t}_{\boldsymbol{i}}-\boldsymbol{t}_{\boldsymbol{0}}\right)$ & $F\left(t_{i}\right)=\left(\frac{i}{N^{\prime}+1}\right)$ & $\ln \left[\ln \left(\frac{1}{1-F\left(t_{i}\right)}\right)\right]$ & Regression \\
\hline 1 & & & & & \\
\hline 2 & 718 & 181.3 & 5.2002 & 0.0625 & -2.7405 & -3.4657 \\
3 & 845 & 540.3 & 6.0875 & 0.1250 & -2.0134 & -1.3572 \\
4 & 912 & 575.3 & 6.3549 & 0.2500 & -1.5720 & -1.0159 \\
5 & 922 & 585.3 & 6.3721 & 0.3125 & -1.2459 & -0.7217 \\
6 & 986 & 649.3 & 6.4759 & 0.3750 & -0.9816 & -0.6808 \\
7 & 1003 & 666.3 & 6.5017 & 0.4375 & -0.5528 & -0.4342 \\
8 & 1027 & 690.3 & 6.5371 & 0.5000 & -0.3665 & -0.3728 \\
9 & 1045 & 708.3 & 6.5629 & 0.5625 & -0.1903 & -0.2275 \\
10 & 1061 & 724.3 & 6.5852 & 0.6250 & -0.0194 & -0.1744 \\
11 & 1085 & 748.3 & 6.6178 & 0.6875 & 0.1511 & -0.0970 \\
12 & 1104 & 767.3 & 6.6429 & 0.7500 & 0.3266 & -0.0374 \\
13 & 1110 & 773.3 & 6.6507 & 0.8125 & 0.5152 & -0.0189 \\
14 & 1278 & 941.3 & 6.8473 & 0.8750 & 0.7321 & 0.4483 \\
15 & 1406 & 1069.3 & 6.9748 & 0.9375 & 1.0198 & 0.7513 \\
\hline \hline
\end{tabular}

Table 2. Regression output for failure data (h) for $\boldsymbol{B}_{4}$ •

\begin{tabular}{lr}
\hline \hline Constant $C$ & -15.8226 \\
Std. Error & 0.4323 \\
R Squared & 0.8445 \\
No. of Observations $N^{\prime}$ & 15 \\
Degree of Freedom & 13 \\
Std. Error of Coefficient & 0.2828 \\
\hline$\beta$ & 2.3762 \\
$\eta$ & 1116.49 \\
$T_{0}$ & 337 \\
\hline \hline
\end{tabular}

12

American Institute of Aeronautics and Astronautics 
Similarly, the other brake assemblies were analyzed. The results are summarized in Table 3 . As indicated earlier, the airplane has four brake assemblies, two on the right $\left(B_{1}\right.$ and $\left.B_{2}\right)$ and two on the left $\left(B_{3}\right.$ and $\left.B_{4}\right)$ as shown in Fig. 2. A comparative assessment of the Weibull reliability parameters of the brake assemblies indicates the following.

1) The minimum guaranteed life $t_{0}$ is in the range from $34.20 \mathrm{~h}$ to $726.75 \mathrm{~h}$.

2) A shape factor $\beta>1$ is observed in each case except the brake assembly $B_{3}$. The values of $\beta$ higher than 1 reflects a time-dependent wear/failure rate or an increasing wear/failure rate of the brake assemblies. The range of $\beta$ observed is from 0.3770 to 2.3762 .

Table 3. Comparison of life of brake assemblies as a function of time.

\begin{tabular}{ccccc}
\hline Brake Assembly & $\boldsymbol{t}_{\boldsymbol{0}}(\mathbf{h})$ & $\boldsymbol{\eta} \mathbf{( h )}$ & $\boldsymbol{\beta}$ & Average Life $\bar{T} \mathbf{( h )}$ \\
\hline$B_{1}$ & 567.80 & 1219.53 & 1.1583 & 1125.36 \\
$B_{2}$ & 726.75 & 1069.76 & 1.2649 & 1025.77 \\
$B_{3}$ & 34.20 & 2621.74 & 0.3770 & 1121.58 \\
$B_{4}$ & 336.70 & 1116.49 & 2.3762 & 1005.27 \\
\hline
\end{tabular}

\section{Results and Comparison}

Evaluating the model adequacy is an important part of any model-building problem. The idea is to examine whether the fitted model is in agreement with the observed data. An informal visual assessment method has been adopted. Figure 10(a) shows a comparison between the actual and the predicted failure rate with respect to time (hours) for $B_{I}$ using artificial neural network and the Weibull model. For the performance evaluation of the ANN and the Weibull regression models, a predictive accuracy of the two models for the given brake assembly data has been compared. For time (hours) input data, Figs. 10(a)-(d) show the actual failure rate, the predicted failure rate from the ANN model, and the predicted failure rate from the Weibull regression model for the four brake assemblies. The results can be considered in two groups (group $A_{1}$ and $A_{2}$ ). Group $A_{1}$ is when the rate of $F\left(t_{i}\right)$, with respect to $\left(t_{i}-t_{0}\right)$, is large at the earlier stage or becomes large after a short time, and/or if there is no major change in the rate of $F\left(t_{i}\right)$ that takes place and remains that way for a longer time, e.g., Fig. 10(a) for the first brake assembly, $B_{1}$. Group $A_{2}$ is when the rate of $F\left(t_{i}\right)$, with respect to $\left(t_{i}-t_{0}\right)$, at the earlier stage is small and remains small for a long time, and/or if there is a major change in the rate of $F\left(t_{i}\right)$ that takes place and remains that way for a long time, e.g., Fig. 10(c) for the third brake assembly, $B_{3}$.

Group $A_{1}$ can be considered as two brake assemblies, i.e., $B_{1}$ and $B_{2}$. Group $A_{2}$ can be considered as two brake assemblies, i.e., $B_{3}$ and $B_{4}$. For group $A_{1}$, the first and second brake assemblies $\left(B_{1}\right.$ and $\left.B_{2}\right)$ are shown in Figs. 10(a) and (b), respectively. For group $A_{2}$, third and fourth brake assemblies $\left(B_{3}\right.$ and $\left.B_{4}\right)$ are shown in Figs. 10(c) and (d), respectively. 


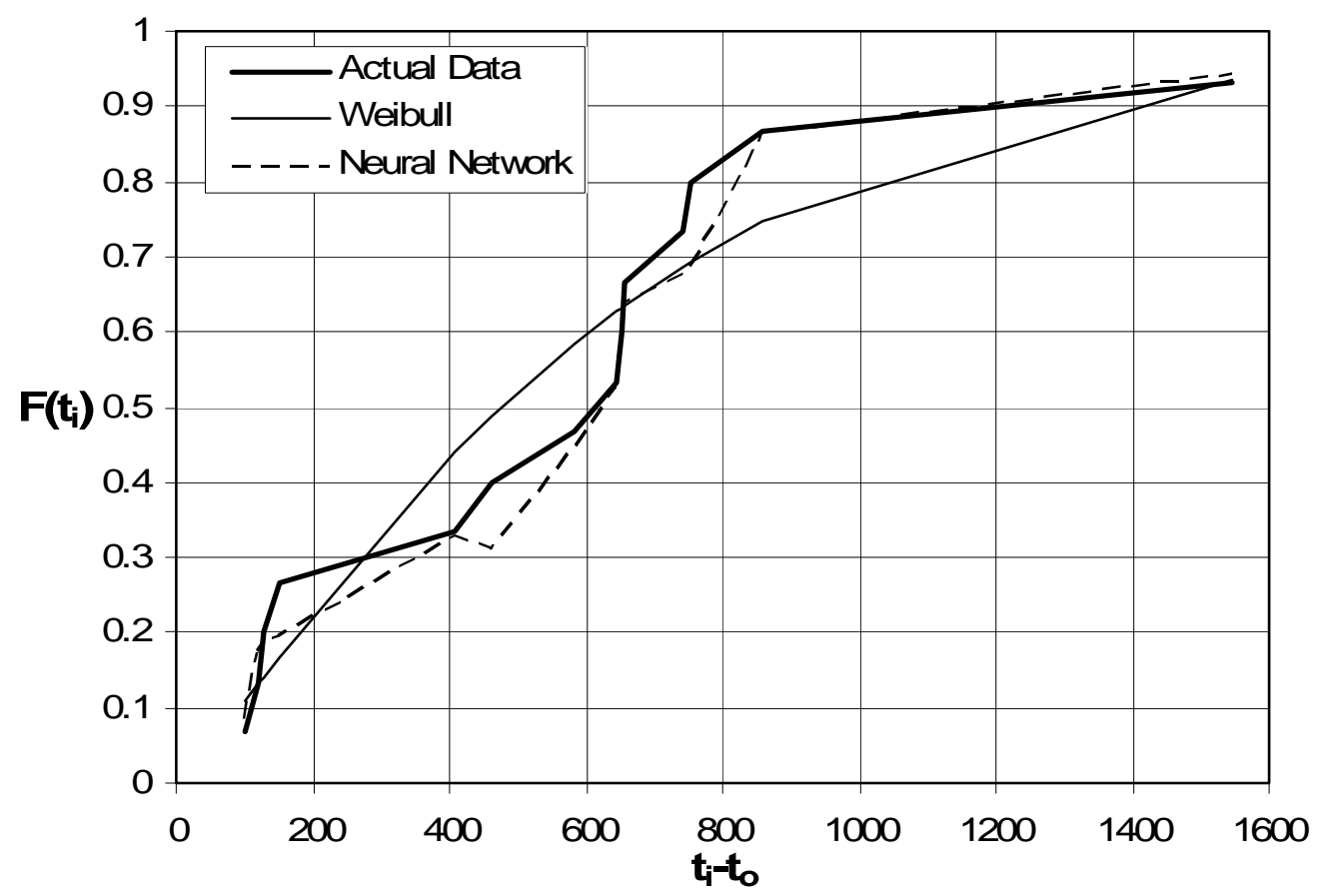

Figure 10(a). Failure rate $F\left(t_{i}\right)$ for Boeing 737 brake assembly $B_{1}$ versus failure data (h) using time parameter.

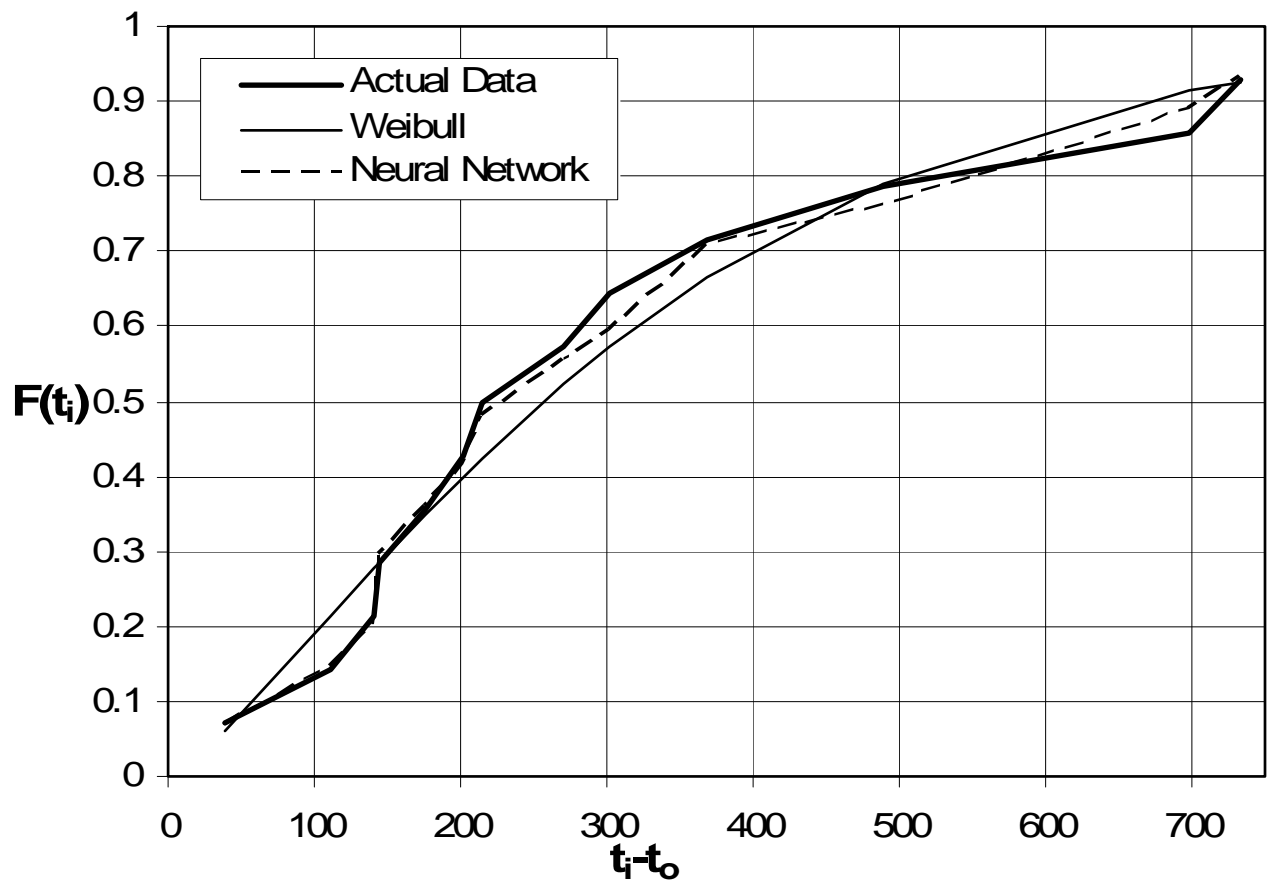

Figure 10(b). Failure rate $F\left(t_{i}\right)$ for Boeing 737 brake assembly $B_{2}$ versus failure data (h) using time parameter. 


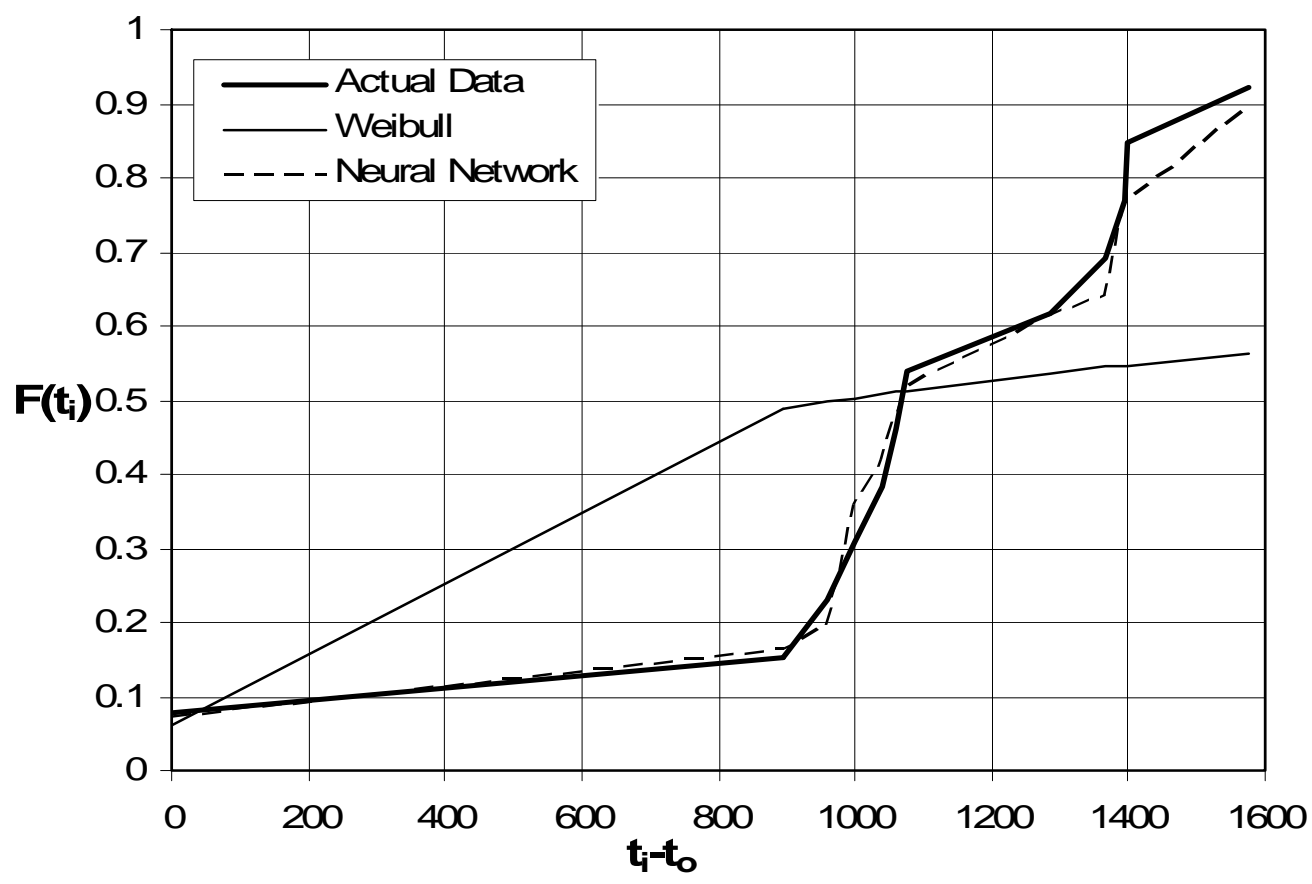

Figure 10(c). Failure rate $F\left(t_{i}\right)$ for Boeing 737 brake assembly $B_{3}$ versus failure data (h) using time parameter.

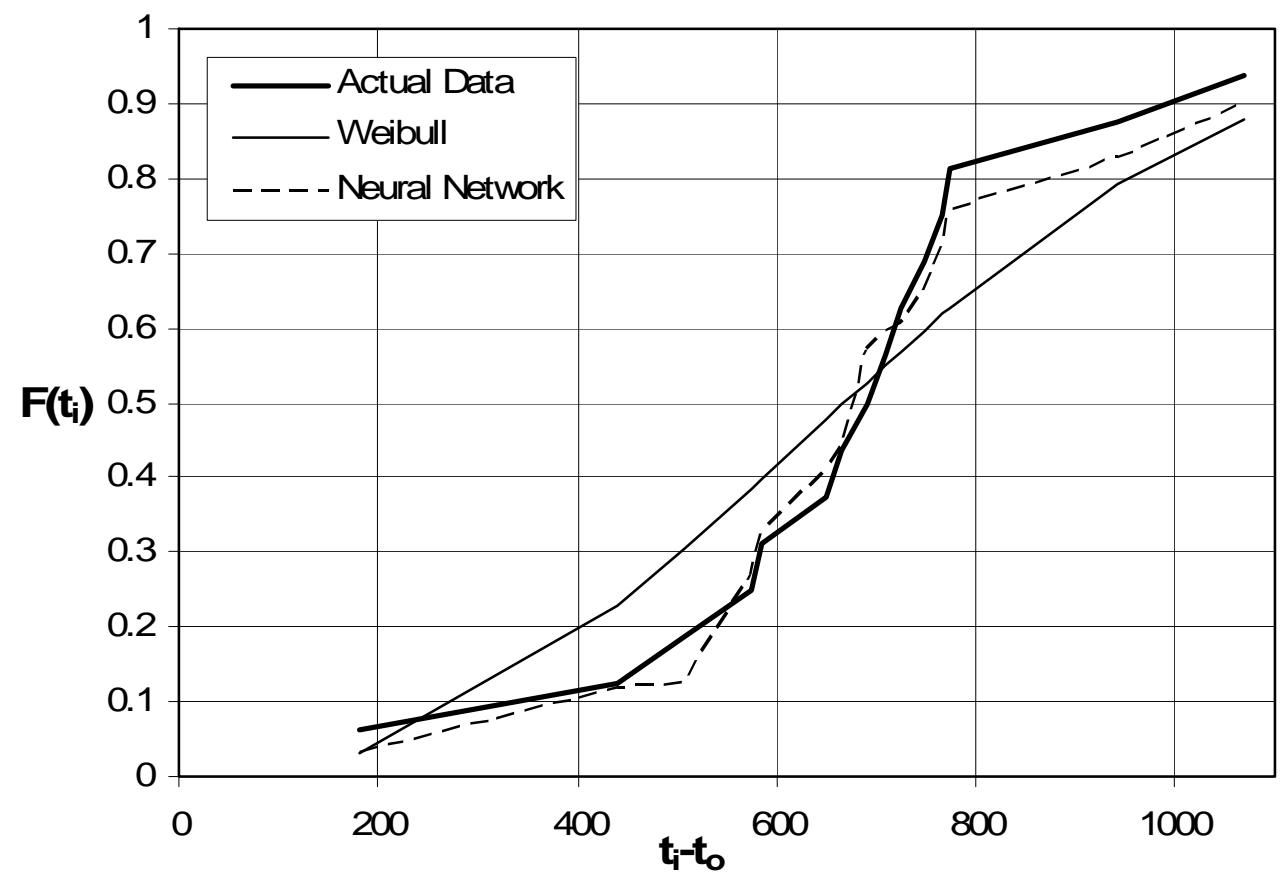

Figure 10(d). Failure rate $F\left(t_{i}\right)$ for Boeing 737 brake assembly $B_{4}$ versus failure data (h) using time parameter. 


\section{Conclusions}

In this study, failure rates of the brake assemblies with respect to time (hours) of four Boeing 737 airplanes are modeled using both artificial neural network and Weibull regression models. A one-layered neural network model is used. A comparative study shows that the three input ANN model performs much better with lesser percentage difference from the actual data than the two and one input models, and six intermediate neurons give much reasonable accuracy than lesser number of intermediate neurons as also verified by visual inspection. With the fact that such comparative analysis finds its applications in various technical and non-technical fields, the results cannot be generalized for all. Hence from the comparison between ANN and Weibull regression models in the present application of failure rate prediction for airplane brake assemblies, it can be concluded that the ANN model predicts better than the Weibull regression model, particularly when the rate of $F\left(t_{i}\right)$ with respect to $\left(t_{i}-t_{0}\right)$ at the earlier stage is small and remains small for a long time, and/or if there is a major change in the rate of $F\left(t_{i}\right)$ that takes place and remains that way for a long time.

Conclusively, the ANN model can be used to schedule a preventive policy for Boeing 737 brake assembly replacement corresponding to an optimal level of brake assembly reliability. To determine logistical support for a specified planning horizon, say for a period of 3 years by determining therein the number of flying hours or landings, one can determine the brake assemblies required during this time and to comparatively assess the quality and performance of the brake assemblies of different manufacturers.

\section{Acknowledgments}

The authors are grateful to the local aviation authority for supplying the data and to King Fahd University of Petroleum and Minerals for supporting this research.

\section{References}

${ }^{1}$ JANE's - All the World's Aircraft 1983-84, Jane's Publishing Company, London, 1983, pp. 329-330.

${ }^{2}$ Boeing 737 Maintenance Manual, Ref. 32-40-08, Boeing, Seattle Washington, 1990, pp. 4.

${ }^{3}$ Al-Garni, A. Z., Sheikh, A. K., and Badar, M. A., "Failure Statistics of Airplane Tires and Reliability-Based Forecasting Strategy," Proceeding of the $4^{\text {th }}$ Saudi Engineering Conference, Vol. 4, KAAU, Jeddah, Saudi Arabia, 1995, pp. 463-469.

${ }^{4}$ Wu, F. Y. and Yen, K. K., "Application of Neural Network in Regression Analysis," IEEE Transactions on Power Systems, Vol. 23, Nos. 1-4, 1992, pp. 93-95.

${ }^{5} \mathrm{Lu}, \mathrm{C} ., \mathrm{Wu}, \mathrm{H}$., and Vemmuri, S., "Neural Network Based Short-Term Load Forecasting," IEEE Transactions on Power Systems, Vol. 8, No. 1, 1993, pp. 336-342.

${ }^{6}$ Al-Garni, A. Z., Sahin, A. Z., and Al-Farayedhi, A. A., "A Reliability Study of Fokker F-27 Airplane Brakes," Reliability Engineering and Systems Safety, Vol. 56, 1997, pp. 143-150.

${ }^{7}$ Al-Garni, A. Z., Ahmed, S. A., and Siddiqui, M., "Modeling Failure Rate for Fokker F-27 Tires Using Neural Network," Transactions of the Japan Society for Aeronautical and Space Science, Vol. 41, No. 131, 1998, pp. $29-37$.

${ }^{8}$ Ganguli, R., Chopra, I., and Has, D., "Helicopter Rotor System Fault Detection Using Physics-Based Model and Neural Network Analysis," AIAA Journal, Vol. 36, No. 6, 1998, pp. 1078-1086.

${ }^{9}$ Bailey, R. A., Pidaparti, R. M., Jayanti, S., and Palakal, M. J., "Corrosion Prediction in Aging Aircraft Materials Using Neural Networks," AIAA/ASME/ASCE/AHS/ASC Structures, in Proceedings of Structural Dynamics and Materials Conference, Vol. 1, No. 111, 2000, pp. 2058-2067.

${ }^{10}$ Pidaparti, R. M., Jayanti, S., and Palakal, M. J., "Residual Strength and Corrosion Rate Predictions of Aging Aircraft Panels: Neural Network Study," AIAA Journal of Aircraft, Vol. 39, No.1, 2002, pp. 175-180.

${ }^{11}$ Al-Garni, A. Z., Jamal, A., Ahmad, A. M., Al-Garni, A. M., and Tozan, M., "Failure-Rate Prediction for De Havilland Dash-8 Tires Employing Neural Network Technique," AIAA Journal of Aircraft, Vol. 43, No. 2, 2006, pp. 537-543.

${ }^{12}$ MuClloch, M. C. and Pitts, W., "A Logical Calculus of the Ideas Imminent in Nervous Activity," Bulletin of Mathematical Biophysics, Vol. 5, 1943, pp. 115-133.

${ }^{13}$ Rosenblatt, F., "The Perception: A Probabilistic Model for Information Storage and Organization in Brain," Psychological Review, Vol. 65, 1958, pp. 386-408.

${ }^{14}$ Minsky, M. L. and Papert, S. A., Perceptrons, The MIT Press, Cambridge, Massachusetts, 1969.

${ }^{15}$ Hopfield, J. J., "Neural Networks and Physical Systems with Emergent Collective Computational Abilities," Proceedings of the National Academy of Science, Vol. 79, 1982, pp. 2554-2558.

${ }^{16}$ Haykin, S., Neural Networks, A Comprehensive Foundation, Prentice-Hall, Englewood Cliffs, New Jersey, 1999.

${ }^{17}$ Werbos, P., "Back-Propagation Through Time: What it Does and How to Do it," Proceedings of IEEE, Vol. 78, No. 10, 1990, pp. 1550-1560.

${ }^{18}$ Martin, R. and Heinrich, B., "Direct Adaptive Method for Faster Back-Propagation Learning: the RPROP Algorithm," Proceedings of IEEE International Conference on Neural Networks, 1993, pp. 586-591. 
${ }^{19}$ De Jesus, O. and Hagan, M. T., "Back-propagation Through Time for a General Class of Recurrent Network," Proceedings International Joint Conference on Neural Networks, Vol. 4, 2001, pp. 2638-2643.

${ }^{20}$ Wang, X. G., Tang, Z., Tamura, H., and Ishii, M., "A modified error function for the back-propagation algorithm, Neurocomputing, Vol. 57, Nos. 1-4, 2004, pp. 477-484.

${ }^{21}$ Kapur, K. C. and Lamberson, L. R., Reliability in Engineering Design, Wiley, New York, 1977.

${ }^{22}$ Sheikh, A. K., Al-Garni, A. Z., and Badar, M. A., "Reliability analysis of airplane tires," International. Journal of Quality and Reliability Management, Vol. 13, No. 8, 1996, pp. 28-38. 